\title{
Helicopter Flight Manoeuvre Statistics via ADS-B: An Initial Investigation Using the OpenSky Network ${ }^{+}$
}

\author{
Joshua Hoole * $\mathbb{D}$, Julian Booker $(\mathbb{D})$ and Jonathan Cooper $\mathbb{D}$ \\ Faculty of Engineering, University of Bristol, Bristol BS8 1TR, UK; j.d.booker@bristol.ac.uk (J.B.); \\ j.e.cooper@bristol.ac.uk (J.C.) \\ * Correspondence: josh.hoole@bristol.ac.uk \\ + Presented at the 9th OpenSky Symposium, Brussels, Belgium, 18-19 November 2021.
}

\section{check for}

updates

Citation: Hoole, J.; Booker, J.D.; Cooper, J.E. Helicopter Flight Manoeuvre Statistics via ADS-B: An Initial Investigation Using the OpenSky Network. Eng. Proc. 2021, 13, 10. https://doi.org/10.3390/ engproc2021013010

Academic Editor: Junzi Sun

Published: 31 December 2021

Publisher's Note: MDPI stays neutral with regard to jurisdictional claims in published maps and institutional affiliations.

Copyright: (C) 2021 by the authors. Licensee MDPI, Basel, Switzerland. This article is an open access article distributed under the terms and conditions of the Creative Commons Attribution (CC BY) license (https:// creativecommons.org/licenses/by/ $4.0 /)$.

\begin{abstract}
Significant challenges exist when defining the usage spectra of helicopter components due to the wide range of missions and manoeuvres flown by helicopters in-service. Automatic Dependent Surveillance-Broadcast (ADS-B) trajectories provide a means of constructing helicopter flight manoeuvre statistics across entire in-service fleets. This paper explores the feasibility of characterising helicopter manoeuvres by applying rule-based algorithms to ADS-B trajectories from a fleet of twin-seat training helicopters. Despite challenges relating to low-altitude ADS-B coverage, a comprehensive set of flight manoeuvre statistics was generated, which highlighted that significant variability exists in helicopter flight manoeuvre occurrences. The generated statistics can also support validation activities concerning design usage spectra assumptions.
\end{abstract}

Keywords: ADS-B; helicopter; fatigue; aircraft trajectories; HUMS

\section{Introduction}

The various mission profiles and manoeuvres flown by helicopters in-service are the most diverse observed within the aerospace sector, leading to significant challenges when conducting the fatigue design of helicopter components [1]. Variability also exists in the manoeuvres flown by the same helicopter type across different flights and operators. Consequently, the definition of accurate usage spectra is a challenging task and standardised spectra have been used previously to support design efforts, such as the well-established Helix and Felix spectra for helicopter rotor blades [2,3]. Whilst standardised spectra are typically derived from in-service load monitoring campaigns, there is the ever-present need to validate the usage spectra assumed during component design with the actual usage spectra that a helicopter is exposed to in-service [4].

'Real-time' tracking of helicopters via Automatic Dependent Surveillance-Broadcast (ADS-B) transponder data and flight trajectories is now a reality [5]. The advantage of characterising usage spectra via ADS-B data is that it can be applied remotely (i.e., no interruption to operations) across entire global fleets, unlike traditional in-service loads monitoring campaigns that are often reliant on a limited number of airframes. It is proposed that ADS-B trajectories could be used to capture in-service helicopter usage spectra, in order to validate previously assumed usage spectra, along with supporting usage spectra development in future design activities. Such activities would lead to more efficient components that retain their integrity in-service and this paper aims to explore the feasibility of constructing helicopter usage spectra using ADS-B trajectories.

\section{Helicopter ADS-B Trajectories}

The utility of helicopter ADS-B trajectories has been considered over the past decade. Assessment of ADS-B signal reception for helicopter flight manoeuvres was performed by Thomas [6], while Gankema and Becher have defined conditions for identifying helicopters in ADS-B datasets [7]. Chin et al. have recently used ADS-B data for altitude and trajectory 
anomaly detection in the helicopter flights of a specific operator [5]. The work presented in the current paper extends this previous work by developing algorithms that identify specific flight manoeuvres within ADS-B trajectories.

A twin-seat primary training helicopter was selected to support this study. This helicopter type was selected due to the fact that larger utility helicopters typically have dedicated flight data recorders and Health and Usage Monitoring Systems (HUMS) [4], whilst such systems are not routinely present on smaller civilian helicopters. Therefore, the selection of a twin-seat training helicopter permits the generation of flight manoeuvre statistics that are not currently available to the aerospace design community.

Within ADS-B trajectories, the relevant data contained within each broadcast (typically one every second) includes the: timestamp, latitude/longitude position, ground speed (metres / second-m/s), track $\left({ }^{\circ}\right)$, vertical speed $(\mathrm{m} / \mathrm{s})$, barometric altitude $(\mathrm{m})$, and GPS altitude $(\mathrm{m})$ [8]. These can be converted to standard aeronautical units: ground speed from $\mathrm{m} / \mathrm{s}$ to knots (knts), vertical speed from $\mathrm{m} / \mathrm{s}$ to feet per minute (fpm) and GPS altitude from $\mathrm{m}$ to feet $(\mathrm{ft})$. An example 3D ADS-B trajectory is shown in Figure 1.

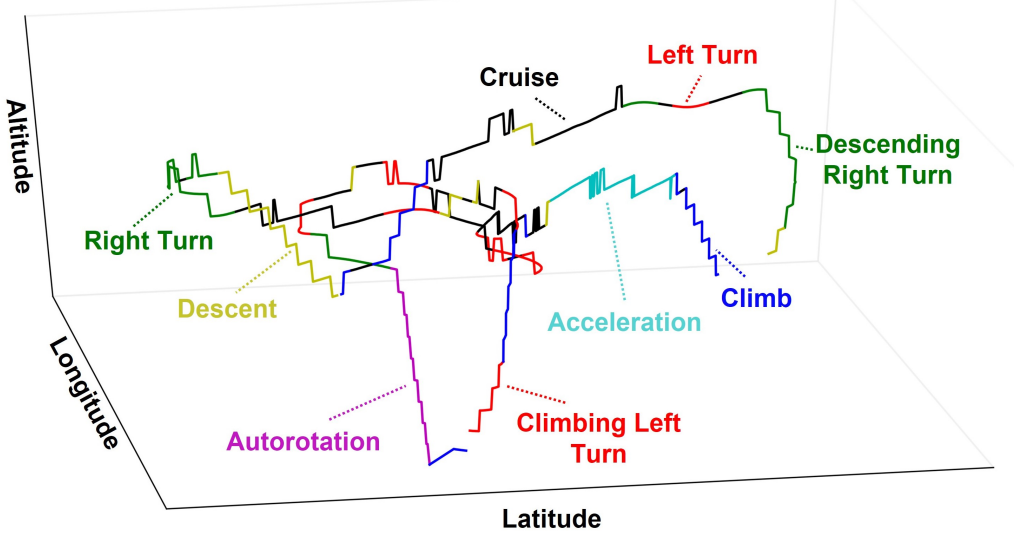

Figure 1. A training helicopter flight ADS-B trajectory with identified manoeuvres labelled.

For the selected helicopter type, the global fleet was reviewed to identify the helicopters that were present within the OpenSky Network database [8]. This investigation identified nine airframes to include within the study, spread across Europe and North America. Using the OpenSky Impala shell, ADS-B trajectories across the period of September 2020 through to August 2021 were extracted for each airframe. Individual flights were then extracted by identifying gaps between trajectory timestamps that were greater than 5 minutes, as also used by the OpenSky Network [8]. Each of the extracted flights were then manually reviewed to identify the quality of the trajectory (i.e., large gaps in the trajectory and clear start and end points) and approximately $30 \%$ of the extracted flights were rejected, leading to a dataset consisting of 2355 flights across the 9 airframes.

Compared to fixed-wing aircraft, helicopters present a unique challenge with respect to flight manoeuvre characterisation due to their ability to travel in a direction (i.e., track) that is significantly different to their heading (i.e., where the helicopter nose is pointing). Consequently, this paper will explore how helicopter manoeuvres can be identified based on the reduced data available in ADS-B trajectories compared to HUMS.

\section{Helicopter Flight Manoeuvre Characterisation Algorithms}

The algorithms used to characterise manoeuvres from each ADS-B trajectory are 'rule-based', where conditional 'if' statements are used to identify manoeuvres based on set thresholds for speed, track, altitude, etc. Rule-based manoeuvre characterisation is sensitive to the selected thresholds and this will be investigated in future studies (see Section 4.1). Olive et al. [9] provide a detailed discussion on the advantages and disadvantages of rule-based approaches. The characterisation algorithm works through the ADS-B 
trajectory on a point-by-point basis and identifies changes in position, track, ground speed, vertical speed, and altitude. The point-to-point characterisations are then grouped with identical consecutive characterisations to extract trajectory 'blocks' representing individual manoeuvres. For the manoeuvre types detailed below, there are additional statistics that could also be collected, including: entry and maximum speeds, turn/yaw rates, altitudes, $\mathrm{climb} /$ descent rates and duration. ADS-B trajectories require pre-processing to remove erroneous data, and whilst the following algorithms were constructed in MATLAB ${ }^{\circledR}$, the reader is directed to the Python traffic package developed by Olive and Basora [10] for ADS-B trajectory pre-processing strategies.

\subsection{Flight Type and Phase Identification: Turns, Climb, Acceleration and Cruise}

Firstly, the overall flight type could be characterised as previously considered by Chin et al. [5]. As this paper concerns a training helicopter, three typical flights were identified: training, cross-country and cross-country with training. A 'cross-country' flight was defined as any flight that had start and end points greater than $10 \mathrm{~km}$ apart. A cross-country flight which featured autorotations (see Section 2.3) was labelled as 'cross-country with training' and all other flights were classified as 'training' flights.

The next stage of trajectory processing requires the identification of different flight phases. It is important to highlight that Chin et al. [11] provide a detailed discussion on identifying flight phases from helicopter flight recorder data and the conditions presented below differ from Chin et al. due to the specific nature of ADS-B trajectories.

Turning manoeuvres were identified via changes in track as a proxy for helicopter heading. Turning manoeuvres are typically composed of several trajectory points, which each show a consistent change in the helicopter track. A minimum turn angle of $20^{\circ}$ was selected to filter out the $\approx 15^{\circ}$ heading deviations observed in straight-flight conditions.

Climbing flight phases were identified as a straight-flight portion in which the minimum observed vertical speed was $250 \mathrm{fpm}$ with a minimum altitude gain of $100 \mathrm{ft}$, based on identified vertical speed and altitude deviations during known straight-flight.

The remaining straight-flight portions in the trajectory were then assessed to identify if the helicopter was accelerating or decelerating and a $15 \mathrm{knt}$ change in helicopter ground speed was required for the registration of an acceleration or deceleration manoeuvre. These thresholds were again set from reviewing straight-flight portions in the trajectories. A deceleration manoeuvre of interest is the helicopter 'quick-stop'. Quick-stops bring the helicopter from a cruise speed (i.e., $>30 \mathrm{knts}$ ) rapidly to a slower speed or hover. From manually reviewing ADS-B trajectories, quick-stop manoeuvres were found to be performed when the observed deceleration rate was greater than $5 \mathrm{knts} / \mathrm{s}$. All remaining straight-flight portions were then labelled as 'cruise' portions.

Turns could then be further characterised by applying the climb/descent and acceleration/deceleration conditional rules. Climb and descent manoeuvres were also further characterised by applying the acceleration/deceleration conditional rules.

\subsection{Hover Manoeuvre Characterisation}

Hover manoeuvres were identified as flight phases with a low ground speed. It was observed within the ADS-B trajectories that the ground speed values became irregular below $5 \mathrm{knts}(\approx 2.5 \mathrm{~m} / \mathrm{s})$. Consequently, any trajectory region where the ground speed was $<5 \mathrm{knts}$ was extracted as a hover phase. The location of the hover manoeuvre could be characterised by identifying clusters of trajectory positions that were within $20 \mathrm{~m}$ (e.g., approximately two rotor diameters) of one another, as shown in Figure 2a.

Helicopters are also able to translate forwards, sideways, and rearwards at slow speed [12] and such manoeuvres occur between consecutive hover locations. Within a hover flight phase, multiple hover locations could be identified by finding the trajectory points that had the greatest number of other trajectory points within the $20 \mathrm{~m}$ threshold (see Figure 2b). Figure 2c shows how hover translation manoeuvres are characterised between two hover locations. It should be noted that this algorithm assumes that the helicopter 
does not change its heading after it enters the first hover location. The consequences of this assumption will be discussed further in Section 4.1.

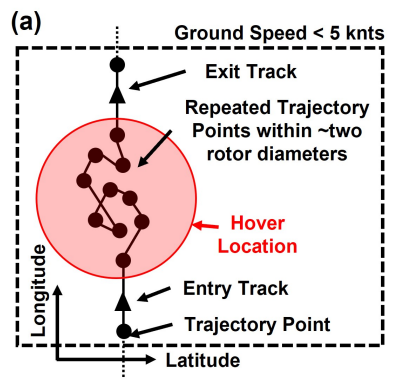

(b)

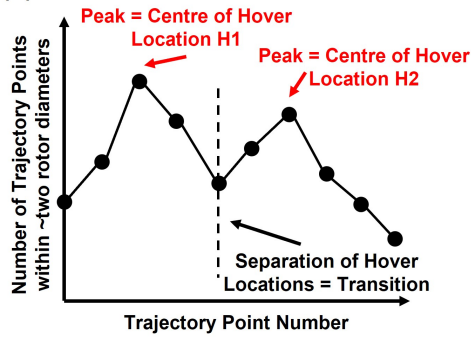

(c)

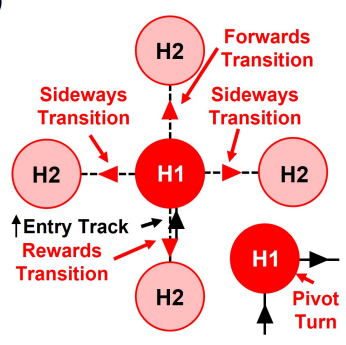

Figure 2. (a) Hover phase identification. (b) Hover location characterisation. (c) Hover manoeuvre characterisation.

For isolated hover manoeuvres, the track entering and leaving the hover were used to identify pivot (i.e., 'on-the-spot') turns, as shown in Figure 2c. It should be noted that changes in track within the hover cannot be used to identify pivot turns as it does not represent helicopter heading changes. Any hover manoeuvres that did not demonstrate a translation, pivot turn, or climb/descent were labelled as a 'stationary' hover.

\subsection{Autorotation Manoeuvre Characterisation}

An 'autorotation' is the manoeuvre performed in response to simulated and real helicopter powertrain failures, with the aim of recovering the helicopter safely to the ground [1]. Autorotations are characterised by high descent rates and are considered a severe fatigue load on helicopter main rotors [1]. From reviewing appropriate helicopter operating manuals, the threshold for identifying an autorotation manoeuvre was any descent flight phase with a maximum descent rate that exceeded $1750 \mathrm{fpm}$.

Autorotation recoveries can take different forms, including straight ahead, an Sturn during the descent, and turns up to and exceeding $360^{\circ}$. Consequently, changes in helicopter track could be used to identify the type of autorotation recovery (e.g., an S-turn recovery would consist of both a left and right turn during autorotation descent).

\subsection{Initial Verification of Algorithms}

Initial verification of the algorithms was performed by processing individual ADS-B trajectories and comparing the resulting characterised manoeuvres as visualised in Figure 1. The limitation of this approach is that there is no prior knowledge of the manoeuvres actually flown. Section 4.1 discusses future planned verification activities.

\section{Helicopter Flight Manoeuvre Statistics}

Each of the 2355 ADS-B trajectories were evaluated using the algorithms to produce manoeuvre statistics, which will be compared to the Helix/Felix training spectra [3] using the values of mode, median, and the mean rounded up to the nearest integer.

Firstly, Figure 3a shows the share of flight types for the training helicopter. As expected, the vast majority of flights were 'training' flights, which are typically expected to incur the most severe usage spectra for helicopters [2]. Figure $3 \mathrm{~b}$ shows the observed duration of flights, which range from minutes (i.e., individual circuits of the airfield) up to three hours. 

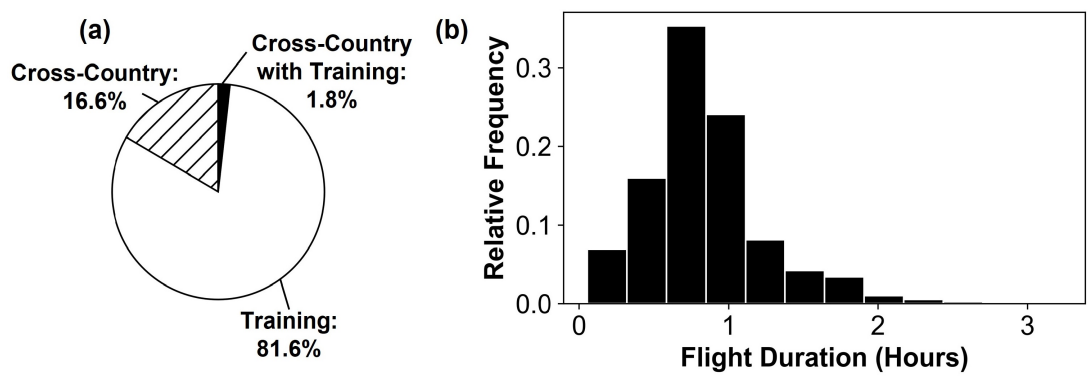

Figure 3. (a) Proportional share of flight types. (b) Variability in flight duration.

Regarding turning manoeuvres, Figure 4 a shows the variability in the number of turning manoeuvres per flight, with a mode of 16 , median of 20, and a rounded average of 22 manoeuvres per flight. Whilst these values are significantly lower than those assumed by the Helix/Felix spectra, the histogram skew highlights that a number of flights exceed the Helix/Felix turn occurrences. Figure $4 \mathrm{~b}$ demonstrates that an equal proportion of left and right turns are performed. The utility of ADS-B data to further characterise manoeuvre types is demonstrated in Figure 4c, where the occurrence of climbing/descending and accelerating/decelerating turns can be identified. The histogram in Figure $4 \mathrm{~d}$ shows the variability in maximum speed observed within each turn and the significance of the bi-modal histogram will be discussed in Section 4 .

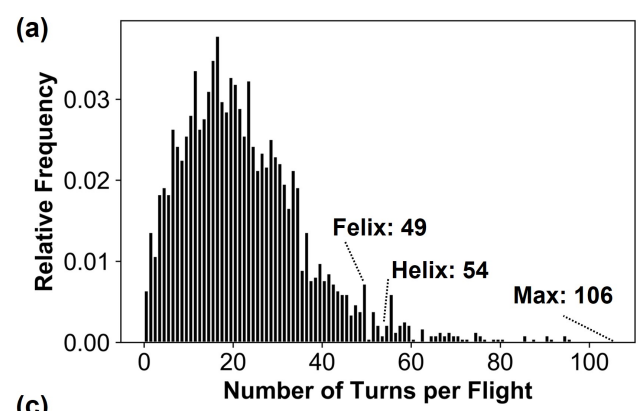

(b)

(c)

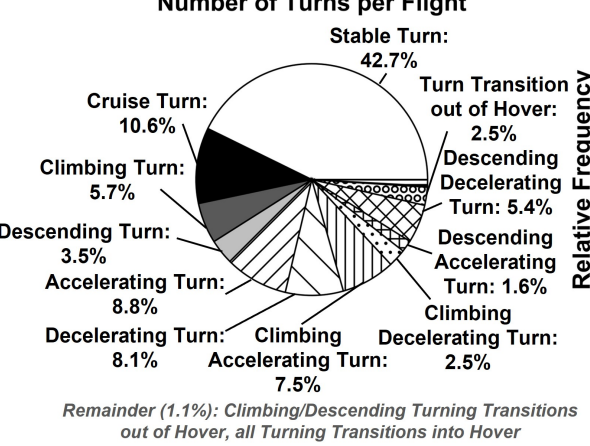

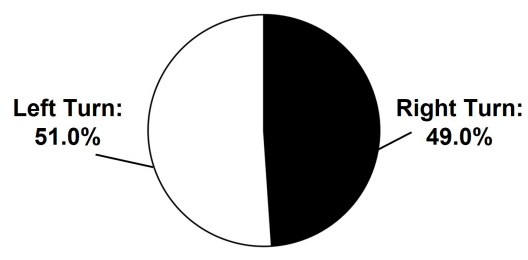

(d)

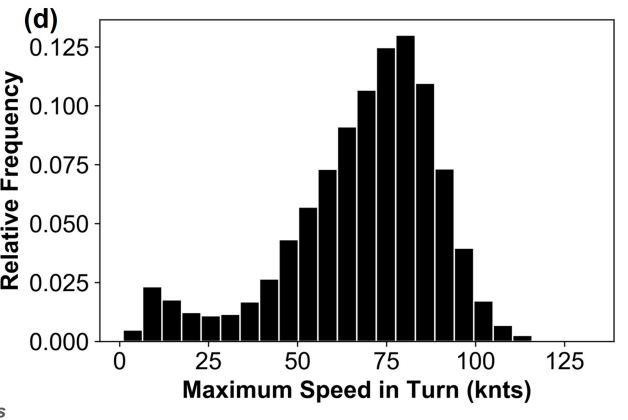

Figure 4. (a) Variability in turning occurrences per flight. (b) Proportional share of left and right turns. (c) Proportional share of turn type. (d) Variability in speed during turn.

The mode number of climbing manoeuvres per flight was found to be 3 with a median of 6 and a rounded average of 8 . Whilst the mode number of climbs is in good agreement with the occurrences assumed in the Helix spectra, the histogram skew in Figure 5a suggests that this may not adequately capture extreme occurrences of climbs for the selected helicopter. Figure $5 \mathrm{~b}$ shows that the majority of climb manoeuvres are performed at a constant speed, potentially reducing the cyclic loads experienced by the helicopter fleet during climbs, as will be considered in Section 4 . 


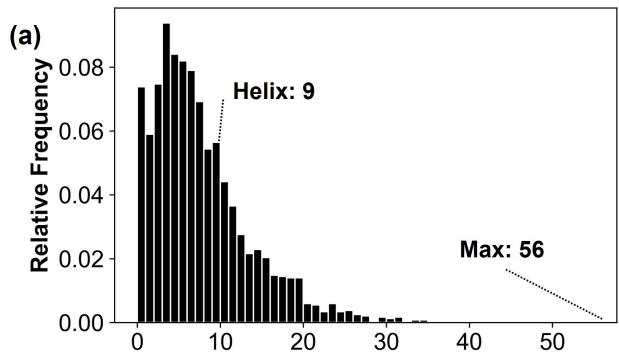

(b)
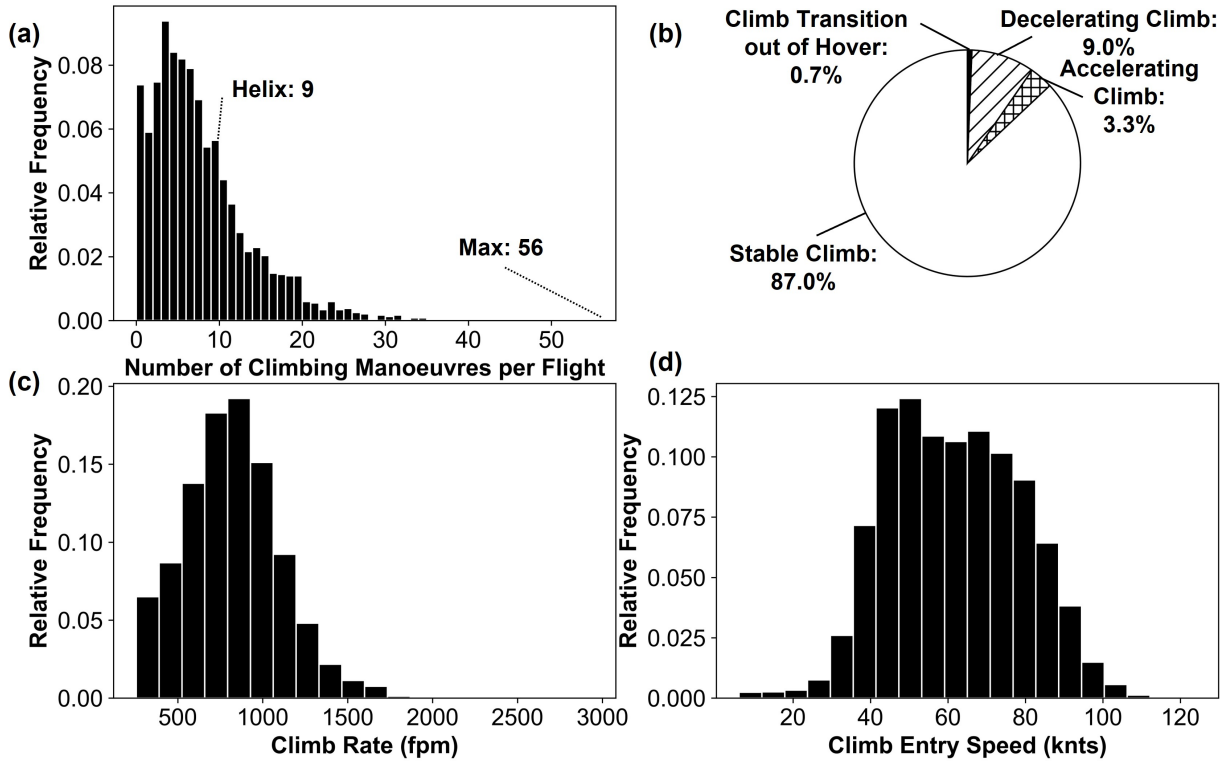

Figure 5. (a) Variability in climbing manoeuvre occurrence. (b) Proportional share of climbing manoeuvre type. (c) Variability in climb rate. (d) Variability in climb entry speed.

Regarding both acceleration and deceleration manoeuvres, the mode occurrence was found to be 1 per flight, with a median of 2, and a rounded average of 3 manoeuvres per flight. Despite the fact that these occurrences are significantly lower than those present within the Helix/Felix spectra, as shown in Figure 6a, the assumption of an equal proportion of acceleration and deceleration manoeuvres has been validated. Figure $6 b, c$ demonstrate that the majority of speed change manoeuvres are related to cruising flight and that only $5 \%$ of flights demonstrate at least one quick-stop.

(a)

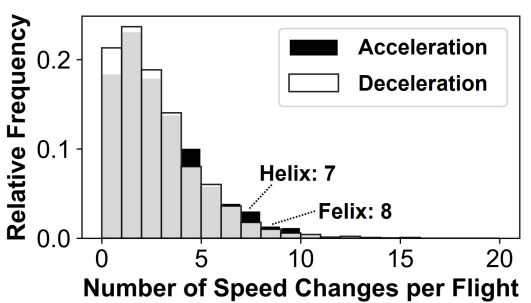

(b)

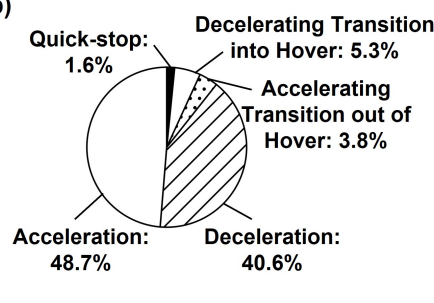

(c)

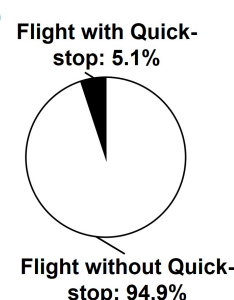

Figure 6. (a) Variability in acceleration/deceleration manoeuvre occurrence. (b) Proportional share of acceleration/deceleration manoeuvre type. (c) Proportion of flights with quick-stop.

Figure 7a shows the scatter in the number of cruise portions observed in the ADS-B trajectories, with the mode number of cruise portions being 15 per flight, with a median of 16 , and a rounded average of 17 . From across Figure $7 b-d$, it is clear that there is significant variability in the altitude, speed, and duration of each cruise phase. The histogram axis in Figure $7 d$ is truncated for clarity and the maximum observed cruise phase duration was approximately $40 \mathrm{~min}$. The bi-modal nature of Figure 7c will be discussed further in Section 4. 
(a)
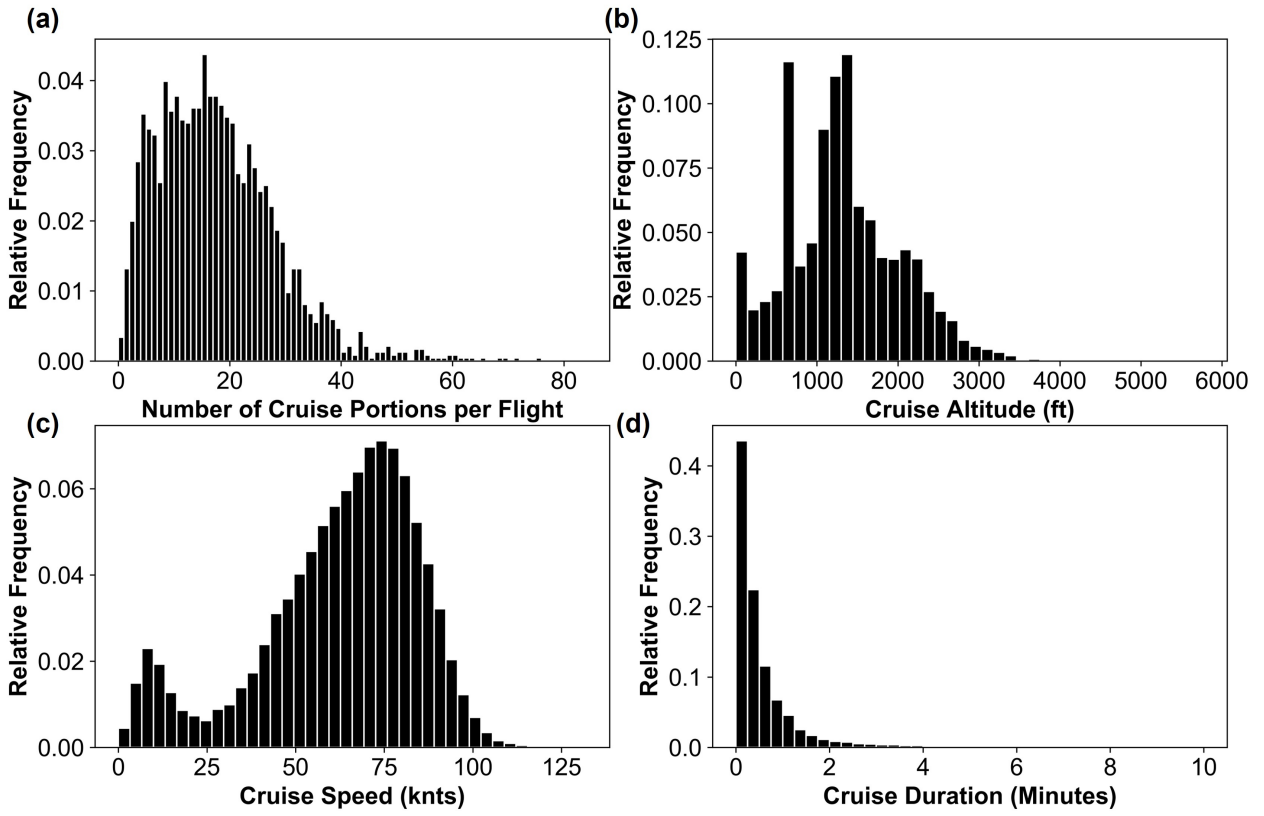

Figure 7. (a) Variability in the number of cruise portions per flight. (b) Variability in cruising altitude. (c) Variability in cruise speed. (d) Variability in cruise duration.

Figure 8a shows the proportion of flights containing at least 1 hover manoeuvre. All helicopter flights would be expected to contain hover manoeuvres during takeoff and landing and consequently, the reason for the limited number of hover manoeuvres being identified in Figure 8a will be discussed in Section 4.1. Figure $8 \mathbf{b}$ shows the number of hover manoeuvres performed in ADS-B trajectories that were observed to have at least 1 hover manoeuvre. The mode number of hover manoeuvres per flight was 1 , with a median of 3 and a rounded average of 5 hover manoeuvres per flight, significantly below the Helix occurrences. Figure $8 \mathrm{c}$ shows that the majority of hover manoeuvres were pivot turns (52\%), with forwards and sideways hover transitions occurring in approximately $5 \%$ of hover manoeuvres. These proportions also disagree with the Helix spectra, where approximately $45 \%$ of hover manoeuvres are stationary, $30 \%$ are pivot turns, $15 \%$ are sideways flight and $10 \%$ involve rearwards flight [3].

(a)

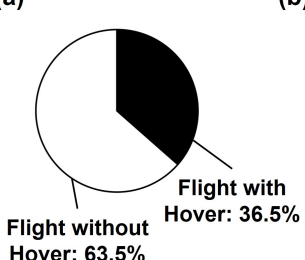

(b)

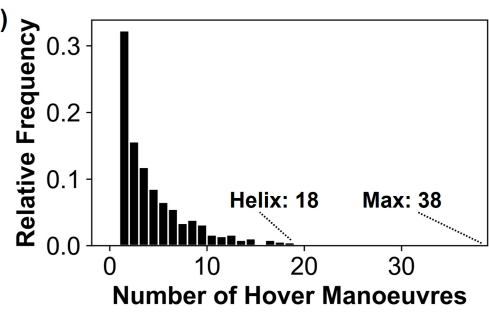

(c)

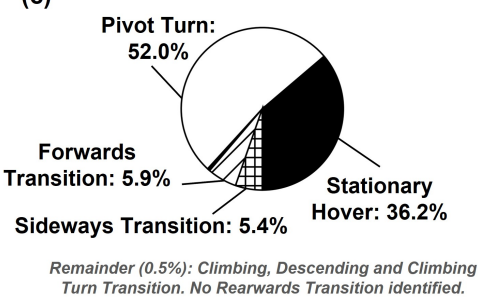

Figure 8. (a) Proportion of flights with observed hover manoeuvres. (b) Variability in hover occurrences for flights with hover manoeuvres. (c) Proportional share of hover manoeuvre type.

Finally, the statistics relating to autorotations are presented in Figure 9. Whilst the proportion of flights performing at least 1 autorotation shown in Figure 9a is lower than the $\approx 50 \%$ of flights suggested in previous work [1], the skew in the histogram shown in Figure 9b highlights that a significant number of flights perform a large number of autorotations. This variability leads to the rounded average of 2 autorotations per flight, which is in good agreement with the Helix and Felix spectra that respectively contain 1 and 3 autorotations per flight. Figure $9 \mathrm{c}, \mathrm{d}$ suggest that the majority of autorotations are straight ahead recoveries that are entered at cruising speed. 
(a)
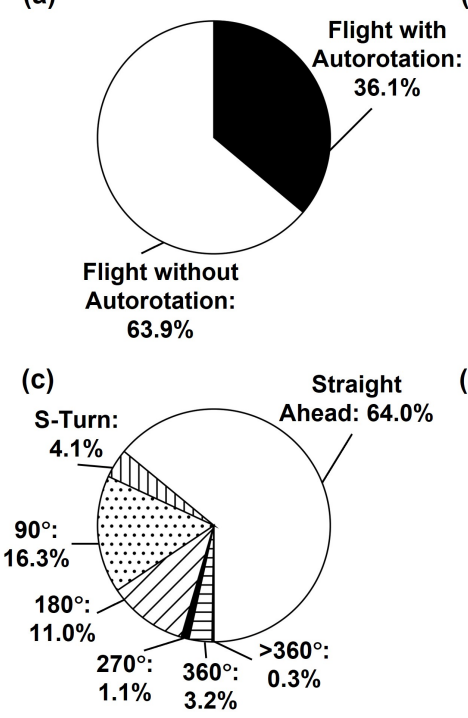

(b)
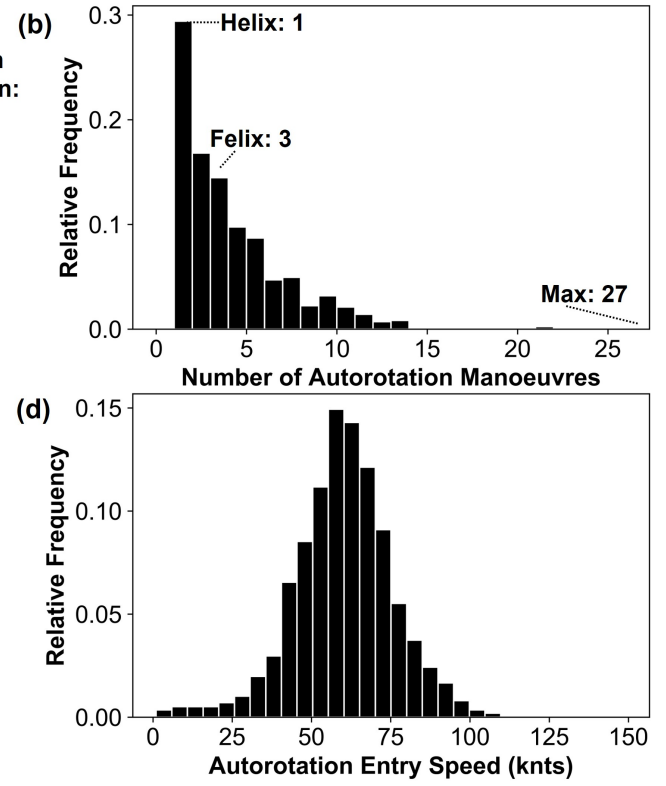

Figure 9. (a) Proportion of flights with autorotation manoeuvres. (b) Variability in autorotation occurrence for flights with autorotations. (c) Proportional share of autorotation recovery type. (d) Variability in autorotation entry speed.

\section{Discussion}

The contents of the previous section have demonstrated that helicopter flight manoeuvre statistics can be generated from ADS-B trajectories. Whilst this paper does not intend to present a final set of manoeuvre statistics, the results provide an indication of how ADS-B data can be used to evaluate helicopter usage spectra for fatigue design.

The exploitation of ADS-B trajectories could permit greater detail to be included within future usage spectra. In the Helix spectra, single turn types are assumed [3]. However, the results from Figure 4c would enable different turn types to be included in usage spectra in proportions reflective of actual usage. Similarly, the bi-modal turn and cruise speeds shown in Figure $4 \mathrm{~d}$,c would enable spectra to reflect that the helicopter operates in slow flight $(\approx 10 \mathrm{knts})$ and cruise $(\approx 75 \mathrm{knts})$ speed conditions.

The results presented in the previous section could also support a challenge of existing spectra. For example, the Helix spectra assumes that all climbing manoeuvres are 'maximum power climbs' [3]. Figure 5c,d show that there is variability in both the climb rate and climb entry speed, suggesting that not every climbing manoeuvre will require maximum power. Consequently, ADS-B derived statistics could support conservatism reduction in design spectra. In addition, autorotations with control reversals are a large low cycle fatigue case for helicopters [2]. However, previous spectra omitted such manoeuvres as they were not considered representative of normal usage [2]. This assumption is supported by the low occurrence of S-turn autorotations, which would feature a longitudinal (i.e., roll) control reversal, shown previously in Figure 9c.

ADS-B trajectories could also be used for the monitoring of individual airframes. For example, Helicopters A and B of the nine airframe fleet were found to operate predominately cross-country flights with only $42.4 \%$ and $6.7 \%$ of their observed flights containing training elements. This is significantly lower than the proportion of training flights $(81.6 \%)$ observed in Figure 3a, suggesting that Helicopters A and B are exposed to a less severe usage spectrum than expected for the fleet. On the other hand, Helicopter $C$ was observed to perform more flights (50.8\% of flights) that contained autorotation manoeuvres than the fleet-level occurrence of $36.1 \%$. This observation suggests that Helicopter $\mathrm{C}$ may be exposed to a more severe usage spectrum. Such information could therefore be used to support life extension activities and HUMS of individual airframes. 
Finally, with the advancement towards urban air mobility it is expected that the inservice loads of helicopters will differ to those previously encountered in helicopter design, due to the adoption of novel helicopter configurations along with hybrid and electric powertrains [13]. As existing standardised spectra are likely to require modification, there is the opportunity to develop new design spectra informed by ADS-B data.

\subsection{Current Limitations and Future Verification Activities}

Despite the utility of ADS-B trajectories discussed above, there are current limitations of the presented methodology that must be addressed within future work.

Firstly, as helicopters often perform slow flight and hovering close to the ground, this can result in manoeuvres within ADS-B trajectories being performed out of the line-ofsight of ADS-B receivers [7]. It is expected that this is the cause of the difference in hover, acceleration, and deceleration occurrences between the Helix/Felix spectra and the results presented in Section 3. It is believed that with the ever increasing coverage and mandated use of ADS-B, this situation will improve in the future. It is also proposed that ADS-B data from the OpenSky Network, with its superior sample rate, could be combined with other ADS-B providers that do capture low altitude manoeuvres.

The other challenge facing the identification of helicopter flight manoeuvres within ADS-B trajectories concerns the fact that only the ground track is available, rather than the helicopter heading. In the presented algorithms, the direction of the helicopter can only be identified entering and leaving the hover. Therefore, should the helicopter perform multiple turns within a hover phase, only a single pivot turn will be identified. Likewise, the algorithms would currently classify a pivot turn-forward hover-pivot turn sequence as sideways flight. Future work will therefore need to identify elements of the ADS-B trajectory that could be used to robustly classify between different types of hover manoeuvres. It is proposed that flight simulation could be used to prototype manoeuvre characterisation algorithms through flying 'known' manoeuvres and identifying specific trajectory characteristics that can be used to identify each manoeuvre type.

Flight simulation could also provide further algorithm verification, by simulating a flight with known manoeuvres leading to a simulated ADS-B trajectory, which could then be evaluated using the algorithms. Ultimately, the use of an ADS-B equipped helicopter to fly a known manoeuvre sequence, or cockpit video recordings with corresponding ADS-B trajectories, would provide the most complete verification of the algorithms.

\section{Conclusions}

The development of usage spectra during the fatigue design of helicopter components is a challenging task due to variability in the manoeuvres that helicopters perform inservice. This paper has explored the use of Automatic Dependent Surveillance-Broadcast (ADS-B) trajectories to evaluate the in-service usage spectra of a training helicopter. Manoeuvre characterisation algorithms were developed and applied to 2355 flights across a helicopter fleet. The resulting manoeuvre statistics were compared with existing standardised spectra. It was identified that in some instances the statistics contradicted the assumed spectra, whilst other cases validated the standardised spectra occurrences. Despite current limitations regarding low-altitude ADS-B coverage and the characterisation of helicopter manoeuvres during hover, it has been shown that ADS-B data provides a route to developing more representative usage spectra for helicopters.

Author Contributions: Conceptualization, J.H., J.B., and J.C.; methodology, J.H.; formal analysis, J.H.; writing - original draft preparation, J.H.; writing — review and editing, J.B. and J.C. All authors have read and agreed to the published version of the manuscript.

Funding: This research received no external funding.

Data Availability Statement: Publicly available datasets were analyzed in this study. The data can be found here: https:/ / opensky-network.org. 
Conflicts of Interest: The authors declare no conflict of interest.

\section{References}

1. Lombardo, D.C. Helicopter Structures-A Review of Loads, Fatigue Design Techniques and Usage Monitoring; Defence Science and Technology Organisation Aeronautical Research Laboratory: Canberra, Australia, 1993.

2. Edwards, P.R.; Darts, J. Standardised Fatigue Loading Sequences for Helicopter Rotors (Helix and Felix), Part 1: Background and Fatigue Evaluation; Royal Aircraft Establishment: Farnborough, UK, 1984.

3. Edwards, P.R.; Darts, J. Standardised Fatigue Loading Sequences for Helicopter Rotors (Helix and Felix), Part 2: Final Definition of Helix and Felix; Royal Aircraft Establishment: Farnborough, UK, 1984.

4. Rustici, S.; Guadalupi, G.; Mariani, U. Implementation of the Health and Monitoring Data for Rotorcraft Fatigue Spectrum. In Proceedings of the European Rotorcraft Forum, Milan, Italy, 12-15 September 2017.

5. Chin, H.-J.; Payan, A.P.; Marvis, D.N.; Johnson, C.C. Knowledge Discovery Within ADS-B Data from Routine Helicopter Tour Operations. In Proceedings of the AIAA Aviation 2020 Forum, Virtual, 15-19 June 2020.

6. Thomas, P. North Sea Helicopter ADS-B/MLat Pilot Project Findings. In Proceedings of the 2011 Tyrrhenian International Workshop on Digital Communications-Enhanced Surveillance of Aircraft and Vehicles, Capri, Italy, 12-14 September 2011; pp. 53-58.

7. Gankema, R.; Becher, O. Spatial Analysis of ADS-B Messages to Identify Rescue Helicopters Using Spark; Large Scale Data Engineering Course (LSDE), Centrum Wiskunde and Informatica (CW\&I): Amsterdam, The Netherlands, 2016.

8. Schäfer, M.; Strohmeier, M.; Lenders, V.; Martinovic, I.; Wilhelm, M. Bringing Up OpenSky: A Large-scale ADS-B Sensor Network for Research. In Proceedings of the 13th IEEE/ACM International Symposium on Information Processing in Sensor Networks, Berlin, Germany, 15-17 April 2014; pp. 83-94.

9. Olive, X.; Sun, J.; Lafage, A.; Basora, L. Detecting Events in Aircraft Trajectories: Rule-Based and Data-Driven Approaches. Proceedings 2020, 59, 8. [CrossRef]

10. Olive, X.; Basora, L. A Python Toolbox for Processing Air Traffic Data: A Use Case with Trajectory Clustering. In Proceedings of the 7th OpenSky Workshop 2019, Zurich, Switzerland, 21-22 November 2019; Volume 67, pp. 73-84.

11. Chin. H.-J.; Payan, A.P.; Johnson, C.; Mavris, D.N. Phases of Flight Identification for Rotorcraft Operations. In Proceedings of the AIAA SciTech 2019 Forum, San Diego, CA, USA, 7-11 January 2019.

12. Liard, F. Helicopter Fatigue Design Guide; AGARD-AG-292; Advisory Group for Aerospace Research \& Development: Neuilly sur Seine, France, 1983.

13. Johnson, W.; Silva, C. NASA concept vehicles and the engineering of advanced air mobility aircraft. Aeronaut. J. 2022, 126, 59-91. [CrossRef] 